\title{
Profile of nelarabine: use in the treatment of T-cell acute lymphoblastic leukemia
}

This article was published in the following Dove Press journal:

OncoTargets and Therapy

6 August 2009

Number of times this article has been viewed

\author{
Kelly M Reilly' \\ David F Kisor ${ }^{2}$ \\ 'Department of Pharmacy Practice, \\ ${ }^{2}$ Department of Pharmaceutical \\ and Biomedical Sciences, Raabe \\ College of Pharmacy, Ohio Northern \\ University, Ada, Ohio, USA
}

\begin{abstract}
Nelarabine is the prodrug of $9-\beta$-arabinofuranosylguanine (ara-G) and is therapeutically classified as a purine nucleoside analog. Nelarabine is converted to ara-G by adenosine deaminase and transported into cells by a nucleoside transporter. Ara-G is subsequently phosphorylated to ara-G triphosphate (ara-GTP), thereby initiating the therapeutic effect by inhibiting DNA synthesis. Nelarabine has been extensively studied in regards to its pharmacokinetics, and the data have demonstrated that ara-GTP preferentially accumulates in malignant T-cells. Clinical responses to nelarabine have been demonstrated in various T-cell malignancies and appear to correlate with a relatively high intracellular concentration of ara-GTP compared to nonresponders. Therefore, this unique drug feature of nelarabine accounts for clinical utilization in treating adult and pediatric patients with relapsed or refractory T-cell acute lymphoblastic leukemia or T-cell lymphoblastic lymphoma. Neuropathy is the most predominant adverse effect associated with nelarabine and the incidence correlates with the dose administered. Myelosuppression has been observed, with thrombocytopenia and neutropenia as the most common hematologic complications. This article reviews the pharmacology, mechanism of action, and pharmacokinetic properties of nelarabine, as well as nelarabine's clinical efficacy in T-ALL, T-LBL, and other hematologic malignancies. The toxicity profile, dosage, and administration, and areas of ongoing and future research, are also presented.
\end{abstract}

Keywords: nelarabine, T-cell acute lymphoblastic leukemia, 9-beta-D-arabinofuranosyl guanine, ara-G

\section{Introduction}

Approximately 4000 cases of acute lymphoblastic leukemia (ALL) are diagnosed annually in the United States with approximately two thirds of the cases being diagnosed in children and adolescents between 3 to 4 years of age, making ALL the most common cancer within this specific population. Current pediatric chemotherapeutic regimens for the treatment of ALL consist of four basic phases in the treatment protocol:remission induction, treatment of clinical or occult centeral nervous system (CNS) disease by consolidation, intensification and maintenance therapy. ${ }^{1}$ Chemotherapeutic regimens consisting of vincristine, glucocorticoid (dexamethasone), and L-asparaginase have successfully induced remission in approximately $95 \%$ of children diagnosed with ALL leading to current long term disease-free survival rates of approximately $80 \%{ }^{2}$

Adults afflicted with ALL experience more difficulty than pediatric patients in terms of disease relapse as current treatment is less successful than observed in the pediatric patient population. Adult chemotherapeutic regimens typically consist of cyclophosphamide, vincristine, dexamethasone or prednisone, L-asparaginase, and an 
anthracycline such as doxorubicin or daunorubicin. ${ }^{1}$ Often methotrexate or cytarabine are used as therapeutic options to treat occult CNS disease. ${ }^{1}$

A challenging and more devastating subset of ALL includes T-cell lymphoblastic leukemia (T-ALL). Children diagnosed with T-ALL account for $10 \%$ to $15 \%$ of the ALL cases and, historically, this form of malignancy has been viewed as extremely difficult to treat and correlates with a poorer overall prognosis. ${ }^{1}$ Adults and pediatric patients with T-ALL have a higher rate of induction failure, early relapse and overt CNS relapse.

Another rare hematologic cancer is lymphoblastic lymphoma (LBL) and accounts for approximately $15 \%$ of non-Hodgkin's lymphomas (NHL). ${ }^{3,4}$ The World Health Organization classifies LBL as a precursor to B-cell/T-cell hematologic malignancies and is considered a nodal or extranodal presentation of ALL. At least $25 \%$ of bone marrow involvement has been considered the differentiating factor between LBL and ALL. The diagnosis of T-cell lymphoblastic lymphoma (T-LBL) is more common and accounts for $80 \%$ of all lymphoblastic lymphomas. ${ }^{4} \mathrm{~T}$-LBL is most commonly observed in children and with an appropriate and intensive chemotherapy regimen anticipated complete remission rates can be approximately $90 \%{ }^{4}$ If patients experience relapsed or refractory cases of LBL they have a poorer prognosis and treatment can become challenging. Therefore advancements in current therapy and management is essential. ${ }^{4}$

Improvements in therapeutic options available for the treatment of T-cell lymphoblastic leukemia and T-cell lymphoblastic lymphoma lead to the investigation and development of 9-beta-D-arabinosylguanine (ara-G) and was innately guided by the current role in therapy and overall success of other purine nucleoside analogs such as cladribine, pentostatin, and fludarabine which are highly utilized in the treatment of various hematologic malignancies. Ara-G was initially synthesized in 1964 and was evaluated ex vivo as an agent that effectively eliminated malignant $\mathrm{T}$ cells found in bone marrow, but poor water solubility and difficulties encountered during drug synthesis significantly limited the drug's use clinically. ${ }^{5-7}$ Through the use of purine arabinonucleoside synthesis, nelarabine was synthesized and is the 6-methoxy derivative of ara-G, having greatly improved solubility making nelarabine a clinically available treatment modality for relapsed or refractory T-cell malignancies.

Nelarabine is a nucleoside prodrug of 9-betaD-arabinofuranosyl guanine (ara-G) and was approved in October 2005 for the treatment of pediatric and adult patients diagnosed with T-cell acute lymphoblastic leukemia (T-ALL) and T-cell lymphoblastic lymphoma (T-LBL) with refractory or relapsed disease following treatment with at least two chemotherapeutic regimens. ${ }^{8,9}$ Nelarabine was granted accelerated approval by the US Food and Drug Administration (FDA) in October of 2005 based on complete and partial response data observed in clinical trials. ${ }^{9}$ In addition, nelarabine received Orphan Drug Designation since the drug demonstrated clinical efficacy in a specific subset of adult and pediatric malignancies, T-ALL and T-LBL. ${ }^{9}$

\section{Pharmacology}

Nelarabine is a substrate for adenosine deaminase and via demethoxylation is converted to ara-G. The conversion of nelarabine to ara- $G$ is necessary to induce the active cytotoxic properties of nelarabine. ${ }^{8}$ Ara-G is transported intracellularly via nucleoside transporters where phosphorylation occurs to eventually convert ara-G to the active nucleotide, ara-GTP. The rate-limiting step is the initial phophorylation of ara- $\mathrm{G}$ to ara-GMP by deoxycytidine kinase and deoxyguanosine kinase. ${ }^{11}$ Triphosphorylation of ara-G to ara-GTP is essential for clinical activity. ${ }^{11}$ It was noted that there is a higher rate of phosphorylation of deoxyguanosine in T cells compared to B cells. Ara-G, an analog of deoxyguanosine, therefore preferentially accumulates in T cells. ${ }^{8}$ Ara-GTP further competes with deoxyguanosine triphosphate in leukemic blast cells for incorporation into DNA. Once ara-GTP has been incorporated into DNA additional DNA synthesis is inhibited, resulting in DNA chain termination and leukemic cell apoptosis. ${ }^{12}$ The specific site of incorporation into the leukemic cell's DNA has not been completely characterized, however it is clear that incorporation into DNA in intact replicating cells is necessary to elicit the therapeutic and cytotoxic effect from nelarabine. ${ }^{12}$ The preferential accumulation in $\mathrm{T}$ cells accounts for the drug's clinical activity in $\mathrm{T}$ cell leukemia and lymphomas and related toxicity profile. ${ }^{13}$

\section{Pharmacokinetics of nelarabine}

The pharmacokinetics of nelarabine have been extensively evaluated and clearly characterized in both adult and pediatric patients with relapsed or refractory hematologic malignancies. ${ }^{8}$ Nelarabine is a water soluble medication and is formulated for intravenous administration. ${ }^{8}$ Pharmacokinetic studies have determined that at the end of a 1 hour infusion of nelarabine concentrations were only slightly higher than concentrations measured at 45 minutes indicating nelarabine was approaching a steady state concentration. ${ }^{8}$ The $\mathrm{C}_{\max }$ and AUC of nelarabine are proportional to the administered dose in the 
range of 5 to $75 \mathrm{mg} / \mathrm{kg} .{ }^{8}$ The concentrations of nelarabine decline with time in a monoexponential fashion, with a halflife of approximately 15 minutes. ${ }^{8}$ There were no statistically significant differences noted in the pharmacokinetics of nelarabine observed in male verses female patients or in patients with various hematologic malignancies including various leukemias. ${ }^{8}$

\section{Pharmacokinetics of ara-G}

Nelarabine is efficiently converted to ara-G, and the $\mathrm{C}_{\max }$ of ara-G occurs at the end of the infusion and is proportional to the dose of nelarabine administered. The AUC of ara-G is proportional to the dose of nelarabine. ${ }^{8}$ Approximately $94 \%$ of the nelarabine dose is metabolically converted to ara-G during a 1 hour infusion and the AUC of ara-G is also proportional to the AUC of nelarabine as one mol of nelarabine yields one mol of ara-G after demethoxylation of nelarabine by adenosine deaminase. ${ }^{8}$ Peak ara-G levels are dependent on the dose of nelarabine administered. ${ }^{8}$

The average clearance of ara-G was found to be approximately $46 \%$ higher in pediatric versus adult patients. The increased clearance of ara-G explains in part the $30 \%$ shorter half-life of ara-G observed in the pediatric patient population compared to the adult population. ${ }^{8}$ The mean half-life for ara-G in pediatric patients was determined to be 2.1 hours as compared to 3 hours in adults. ${ }^{8}$ The clearance of ara-G is weakly associated with creatinine clearance and the dose does not need to be adjusted based on renal function. ${ }^{8}$ No significant differences were noted in volume of distribution in pediatric and adult patient populations. ${ }^{8}$ Ara-G has a relatively short half-life in both adult and pediatric populations, and time for elimination (greater than 5 half-lives) of ara-G was sufficient to avoid accumulation after daily 1 hour infusions of nelarabine when administered for 5 consecutive days. ${ }^{8}$ The concentration of ara-G measured on days 2 through 5 during a once daily infusion were comparable and did not suggest accumulation over a 5-day regimen. ${ }^{8}$ Concentrations and pharmacokinetic parameter values of ara-G were evaluated in patients with different diagnoses, and were determined to be similar in patients with different hematologic malignancies. ${ }^{8}$ Related pharmacokinetic data are presented in Table 1.

\section{Pharmacokinetics of ara-GTP}

A range of doses of nelarabine were administered to 19 patients (both adult and pediatric) with hematologic malignancies and leukemic cell concentrations of ara-GTP were determined. The ara-GTP median $\mathrm{C}_{\max }$ values were
Table I Pharmacokinetic parameter values (mean \pm SD) for nelarabine, ara-G, ara-GTP5,8,14

\begin{tabular}{|c|c|c|c|}
\hline & Pediatric & Adult & \\
\hline \multicolumn{4}{|l|}{ Nelarabine } \\
\hline $\mathrm{Vd}(\mathrm{L})$ & $8.511 \pm 11.518$ & $4.197 \pm 5.802$ & $N S^{a}$ \\
\hline $\mathrm{CL}(\mathrm{L} / \mathrm{kg} / \mathrm{h})$ & $9.259 \pm 12.794$ & $5.875 \pm 8.434$ & $N S^{a}$ \\
\hline$t_{1 / 2}$ (minutes) & $14.1^{b}$ & $16.5^{\mathrm{b}}$ & $\mathrm{NS}^{\mathrm{a}}$ \\
\hline \multicolumn{4}{|l|}{ ara-G } \\
\hline $\mathrm{Vd}(\mathrm{L})$ & $1.023 \pm 0.345$ & $0.923 \pm 0.231$ & $N S^{a}$ \\
\hline$C L(L / k g / h)$ & $0.312 \pm 0.112$ & $0.213 \pm 0.072$ & $P<0.0001$ \\
\hline$t_{1 / 2}(h)$ & $2.1^{b}$ & $3.0^{\mathrm{b}}$ & $P<0.0 \mathrm{I}$ \\
\hline \multicolumn{4}{|l|}{ ara-GTP } \\
\hline \multirow[t]{2}{*}{ Mean ara-GTP $(\mu \mathrm{M})$} & & $435 \pm 519$ & \\
\hline & & $746 \pm 872^{c}$ & \\
\hline$t_{1 / 2}(h)$ & $>24^{\mathrm{b}, \mathrm{d}}$ & & \\
\hline
\end{tabular}

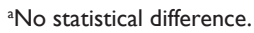

bHarmonic mean value.

'Nelarabine following fludarabine (combination)

dPatient with T-cell disease.

Abbreviations: CL, clearance; $\mathrm{Vd}$, volume of distribution.

$23,42,85$, and $93 \mu \mathrm{mol} / \mathrm{L}$ after administration of nelarabine doses of $20 \mathrm{mg} / \mathrm{kg}, 30 \mathrm{mg} / \mathrm{kg}, 40 \mathrm{mg} / \mathrm{kg}$, and $60 \mathrm{mg} / \mathrm{kg}$. ${ }^{14}$ Patients diagnosed with T-cell lymphoblastic leukemia had the highest leukemic cellular concentrations of ara-GTP ( $\mathrm{n}=7$; median $140 \mu \mathrm{mol} / \mathrm{L})$ in comparison to other hematologic malignancies $(n=9$; median $50 \mu \mathrm{mol} / \mathrm{L})$. Accumulation of ara-GTP was found to be greatest in T-lymphoblastic cells compared to mononuclear cells $(\mathrm{n}=3$; median $30 \mu \mathrm{mol} / \mathrm{L})$. Overall retention of ara-GTP was the greatest in T-leukemia cells compared to other types of leukemic cells (11 out of the 19 patients). ${ }^{14}$ Cellular pharmacokinetics demonstrate and support the concept that ara-GTP accumulates in a dose dependent fashion. ${ }^{14}$ The linear relationship between ara-G concentrations and the varying dose of nelarabine correlated with ara-GTP accumulation. ${ }^{14,16}$ This has been demonstrated both in vitro and in vivo. ${ }^{14,16}$

Clinical efficacy of nelarabine and cellular pharmacokinetics of ara-GTP were recently evaluated in a phase I trial directed by Gandhi et al with 35 patients diagnosed with indolent leukemias (B-cell chronic lymphocytic leukemia and T-cell prolymphocytic leukemia). ${ }^{17}$ Cellular concentrations and evidence of ara-GTP accumulation were evaluated in 25 of the original 35 patients for the first cycle of treatment on days 1 through 2 of three different protocols. ${ }^{17}$ The following protocols were defined as: schedule A administered nelarbine 20 to $60 \mathrm{mg} / \mathrm{kg} /$ day for 5 days, schedule B administered nelarabine $1.5 \mathrm{~g} / \mathrm{m}^{2}$ to $2.9 \mathrm{~g} / \mathrm{m}^{2}$ on days 1,3 and 5 and schedule $\mathrm{C}$ administered nelarabine $1.2 \mathrm{~g} / \mathrm{m}^{2}$ on days 1 , 3 and 5 with fludarabine $30 \mathrm{mg} / \mathrm{m}^{2}$ administered 4 hours 
prior on days 3 and $5 .{ }^{17}$ The median peak concentration for ara-GTP was determined to be $89 \mu \mathrm{mol} / \mathrm{L}$ with a range of 22 to $1438 \mu \mathrm{mol} / \mathrm{L} .{ }^{17}$ In order to account for the differences in dose and the potential effect on ara-GTP leukemic concentration the doses were adjusted and the median peak values of ara-GTP were $65 \mu \mathrm{mol} / \mathrm{L}$ (range 17 to $1438 \mu \mathrm{mol} / \mathrm{L}$ ) which was ultimately found to be similar to the unadjusted values. ${ }^{17}$ The similarity in ara-GTP concentrations and ranges determined after dose-adjustment suggests that the leukemia cells may have an intrinsic ability to accumulate ara-GTP and that the ara-GTP concentration is not strictly defined by the dose administered ${ }^{17}$ Further measures were taken to determine the retention of ara-GTP after peak araGTP concentrations. Samples of peripheral leukemic cells were obtained from 13 patients and 1 patient diagnosed with T-cell prolymphocytic leukemia (T-PLL) demonstrated that the level of ara-GTP became level at 12 hours and the level of ara-GTP was sustained for an additional 36 hours. ${ }^{17}$ Cellular samples evaluated after the second and third infusion showed a continual increase in leukemic concentrations of ara-GTP. ${ }^{17}$ The median $\mathrm{t}_{1 / 2}$ was determined to be greater than 24 hours (range 9 to $>42$ hours) and in T-PLL and BCLL the $t_{1 / 2}$ was similar and the range was 12 to $>42$ hours and 9 to $>42$ hours, respectively. ${ }^{17}$ Accumulation and retention of ara-GTP was determined to be relatively similar among the different treatment protocols. ${ }^{17}$

\section{Pharmacokinetic parameters with co-administration of fludarabine and nelarabine}

A pilot study combining nelarabine and fludarabine was designed to evaluate the biochemical modulating effects of fludarabine on cellular metabolism of ara-GTP and the relationship between clinical response and accumulation ara-GTP in leukemic cells. ${ }^{5}$ The presumption was the co-administration fludarabine with nelarabine would augment intracellular concentration of ara-GTP in leukemic cells that do not preferentially accumulate therapeutic concentrations of the active triphosphate such as demonstrated in B-cell lymphoblasts. ${ }^{5,14}$ This concept was first eluded to in an earlier study by Gandhi et al where the majority of clinical responses secondary to nelarabine were observed in patients diagnosed in T-cell lymphoblastic malignancies, however a single patient diagnosed with B-cell chronic lymphocytic leukemia (B-CLL) achieved a partial remission. ${ }^{14}$ The patient diagnosed with B-CLL had received prior treatment with combination cyclophosphamide and fludarabine and then received treatment with nelarabine therefore achieving greater leukemic concentrations of ara-GTP than other study participants diagnosed with B-cell malignancies. ${ }^{14}$

The rationale supporting the coadministration of fludarabine in conjunction with nelarabine is the direct inhibition of ribonucleotide reductase by fludarabine, resulting in increased ara-GTP concentrations as reflected in Table 1. It was noted by Gandhi et al that the increased ara-GTP concentrations in the face of fludarabine may be affected by residual ara-GTP from previous nelarabine doses. ${ }^{5}$ With the accumulation of fludarabine and fludarabine triphosphate (F-ara-ATP) there could be an overt decrease in deoxynucleotides, including deoxycytidine triphosphate and deoxyguanosine triphosphate. The overall decrease in deoxynucleotides was hypothesized to result in a decreased feedback inhibition of deoxynucleotide kinases including deoxycytidine kinase and deoxyguanosine kinase. Both of these kinases are involved in the initial phosphorpylation of ara-G to ara-GMP and would therefore lead to an increase in the phophorylation rate of ara-G to ara-GMP. This has been determined to be the ratelimiting step in the phosphorylation cascade. ${ }^{11}$

Nelarabine at a dose of $1.2 \mathrm{~g} / \mathrm{m}^{2}$ was administered intravenously on days 1,3 and 5 . Fludarabine $\left(30 \mathrm{mg} / \mathrm{m}^{2}\right)$ was administered intravenously over 30 minutes on days 3 and 5 , four hours prior to the nelarabine infusion. ${ }^{5}$ Samples of plasma and cellular concentrations were collected during the initial 5 days in order to detect any differences with the two agents being coadministered. ${ }^{5}$

During the combination therapy of fludarabine and nelarabine the pharmacokinetics of ara-G were assessed on day one of the five day regimen, when nelarabine was administered as a single agent, and on a subsequent day when combined with fludarabine. ${ }^{5}$ The concentrations of ara-G when nelarabine was administered alone or in combination with fludarabine was similar as was the half-life of ara-G. ${ }^{5}$ The day 1 ara-G $\mathrm{C}_{\max }$ and $\mathrm{AUC}_{0 \text {-infinity }}$ were not different than day 3 values. Gandhi et al concluded that when fludarabine was administered 4 hours prior to nelarabine, there was no significant effect on the concentrations and pharmacokinetics of ara-G.

Cellular retention and accumulation of ara-GTP levels were evaluated on days one through four. ${ }^{5}$ The ara-GTP leukemic cell elimination half-life $\left(\mathrm{t}_{1 / 2}\right)$ was evaluated after 48 hours. It was determined that a $t_{1 / 2}$ greater than 35 hours resulted in concentrations being $45 \%$ to $100 \%$ of peak ara-GTP concentrations for a prolonged period, compared to patients with a more rapid ara-GTP elimination, with a $\mathrm{t}_{1 / 2}$ equal to 18 to 27 hours resulting in concentrations being $17 \%$ to $27 \%$ of peak ara-GTP concentrations. It was determined that after the first and second nelarabine infusion the 
ara-GTP $\mathrm{t}_{1 / 2}$ was similar. ${ }^{5}$ Specific ara-GTP pharmacokinetics were compared to data obtained on day three after the nelarabine and fludarabine infusion. There was an observed increased peak in ara-GTP concentrations on day 3 compared to day 1; however it was difficult for the investigators to fully attribute this observation to the modulating effects of fludarabine of ara-GTP metabolism. There was accumulation of ara-GTP found in the majority of patient cases which were attributable to previous infusions, with the residual percent ranging from $17 \%$ to $100 \%$ of the peak value found in leukemia cells. Pharmacokinetic parameters for nelarabine before and after administration with fludarabine were similar to previously reported values. Fludarabine does not influence an inhibitory effect on adenosine deaminase, so the initial step in drug conversion to the active component, ara-G, would not be expected to be altered.

\section{Pharmacodynamics}

Clinical response to nelarabine has been found to be independent of the dose of nelarabine administered. ${ }^{18}$ Gandhi et al characterized the cellular accumulation of ara-GTP and the related clinical response elicited in patients diagnosed with various hematologic malignancies including T-ALL, CML-BC (chronic myeloid leukemia, in blast crisis) and B-cell chronic lymphocytic leukemia. Within the study patients with hematologic malignancies received 20 to $60 \mathrm{mg} / \mathrm{kg}$ of nelarabine for 5 consecutive days. The $\mathrm{C}_{\max }$ of ara-GTP was evaluated and determined to occur at the end of the infusion in 7 patients, 2 hours post-infusion in 8 patients and 4 hours post-infusion in the remaining 4 patients. ${ }^{14}$ Patients who experienced a response to treatment with nelarabine accumulated significantly higher levels of ara-GTP compared to patients that failed to have a clinical response. The median ara-GTP concentration observed in 7 responding patients diagnosed with T-ALL, CML-BC, B-CLL was $140 \mathrm{micromol} / \mathrm{L}$ versus a concentration of 44 micromol/L in the 9 nonresponders. ${ }^{14}$

\section{Clinical efficacy}

The primary use of nelarabine is for the treatment of refractory hematologic malignancies. In a phase I study, nelarabine (506U78) was administered to patients with refractory hematologic malignancies in relapse to determine the maximum tolerated dose, related toxicity, and pharmacokinetic parameter estimates of nelarabine. The study included 93 patients who received 1 to 16 cycles of nelarabine as a 1 hour infusion daily for 5 consecutive days every 21 to 28 days. ${ }^{18}$ Patients were stratified into 4 groups: adult leukemia ( $\leq 75$ years of age), pediatric leukemia ( $\leq 21$ years of age), adult lymphoma and pediatric lymphoma. Dose escalation of nelarabine began at $5 \mathrm{mg} / \mathrm{kg} /$ dose and was directed by protocol to increase doses to $10,20,40,60,75$ and $90 \mathrm{mg} / \mathrm{kg} /$ dose. $^{18}$ The protocol was amended due to toxicity encountered at $75 \mathrm{mg} / \mathrm{kg} /$ dose level. ${ }^{18}$ The amended protocol evaluated the adult and pediatric dose of nelarabine $1.2 \mathrm{gm} / \mathrm{m}^{2} /$ day which is equivalent to an adult dose of $40 \mathrm{mg} / \mathrm{kg}$ and a pediatric dose of $60 \mathrm{mg} / \mathrm{kg}$. Of the 93 patients enrolled 59 were adults and 34 were children. ${ }^{18} \mathrm{~A}$ total of $66 \%$ of patients had T-cell malignancies, $22 \%$ had B-cell malignancies, and 13\% had myeloid or other hematologic malignancies. The majority of patients enrolled in the study experienced treatment failure with at least 2 prior therapies for their disease with a median number of 3 prior regimens. ${ }^{18}$ Twenty-seven patients received a previous bone marrow transplant, 39 patients received total body irradiation, 14 received cranial or craniospinal irradiation and 45 received intrathecal chemotherapy for treatment of disease within the CNS. Thirty-nine patients (42\%) were diagnosed with T-lymphoid disease including acute lymphoblastic leukemia or lymphoblastic lymphoma.

In T-lymphoid lineage malignancies ( $\mathrm{T}$-cell acute lymphoblastic leukemia and T-cell lymphoblastic lymphoma) a total of $23 \%(n=9 / 39)$ of both adult and pediatric patients achieved a complete response. In the pediatric subgroup, $27 \%$ $(\mathrm{n}=7 / 26)$ of the patients had a complete response and $15 \%$ $(n=2 / 13)$ of adults had a complete response. ${ }^{18}$ Of the $23 \%$ of complete responders 2 patients were removed from the study to pursue a second bone marrow transplant, with 1 of these patients surviving in complete remission 4 years after mismatched cord blood transplantation. ${ }^{18}$ The other patient died in remission secondary to graft-versus-host disease (GVHD) 3 months post-transplantation. Partial responses were observed in 31\% (12/39) of both adult and pediatric populations with $71 \%(n=15 / 21)$ responders having experienced 2 or more relapses and $48 \%(n=10 / 21)$ having had treatment failure with a prior bone marrow transplantation. ${ }^{18}$ Complete responses and partial responses observed within the T-lymphoid lineages occurred at multiple dose levels of nelarabine. Another patient with chronic myeloid leukemia in lymphoid blast crisis that had experienced treatment failure with 2 prior induction regimens prior to study enrollment achieved a complete response for 57 weeks and then experienced disease relapse within the CNS. ${ }^{18}$

Responses were seen in 30\% (3/10) of patients with $\mathrm{T}$ - or B-cell lineage CLL (partial remission). ${ }^{18}$ One patient with prolymphocytic leukemia experienced hematologic 
improvement after receiving treatment during the study with nelarabine. Patients considered being refractory to other nucleoside analogs including cytarabine, fludarabine and pentostatin responded to treatment with nelarabine. ${ }^{18}$

The maximum tolerated dose of nelarabine administered daily over 1 hour for 5 consecutive days was determined to be $60 \mathrm{mg} / \mathrm{kg} /$ dose for children and $40 \mathrm{mg} / \mathrm{kg} / \mathrm{dose}$ for adults. ${ }^{18}$ When evaluating the overall results of the study across all diagnoses and both adult and pediatric populations $(n=93)$, $11 \%(n=10)$ achieved a complete response, $20 \%(n=19)$ had a partial response and $49 \%(n=46)$ had a hematological improvement, stable disease, no response or disease progression. ${ }^{18}$ The remaining $19 \%(\mathrm{n}=18)$ of the patient population were considered nonassessable for response due to: hypocellular bone marrow $(n=1)$, incomplete bone marrow/tumor evaluation at maximal clinical response $(n=7)$, death before 21 days (not attributable to the study drug) $(n=9)$ and removal from the study secondary to adverse events before 21 days $(\mathrm{n}=1)$. $^{18}$

In summary, complete and partial responses were observed in $54 \%(n=21 / 39)$ of both adult and pediatric patients with T-cell acute lymphoblastic leukemia or T-cell acute lymphoblastic lymphoma after 1 to 2 courses of therapy. ${ }^{18}$

Another trial conducted by the Cancer and Leukemia Group B evaluated the efficacy and safety of nelarabine in patients diagnosed with either T-cell acute lymphoblastic leukemia (T-ALL) or T-cell lymphoblastic lymphoma (T-LBL). The patient population consisted of 40 patients with a total of 39 receiving at least 1 dose of nelarabine. The population comprised 32 males and 7 females, ranging in age from 16 to 66 years with a median age of 34 years. ${ }^{15}$ A total of 26 patients were diagnosed with T-ALL and 13 with T-LBL. All patients enrolled were refractory or had relapsed to at least 1 induction regimen after achieving a complete response to prior chemotherapy treatment. The majority of the patient population had an Eastern Cooperative Oncology Group (ECOG) performance status of $0(n=11)$ or $1(\mathrm{n}=17) .{ }^{15}$ Both groups of patients received nelarabine $1.5 \mathrm{~g} / \mathrm{m}^{2}$ on days 1,3 and 5 and repeated this regimen for a total of 2 induction cycles. ${ }^{15}$ The patients who achieved a complete remission after receiving treatment with nelarabine were permitted to receive an additional 2 courses of therapy. ${ }^{15}$

From the initial 40 patients enrolled, 39 patients were evaluated after receiving at least 1 treatment dose of nelarabine with 26 patients $(67 \%)$ having T-ALL and 13 patients (33\%) having T-LBL. ${ }^{15}$ A total of $31 \%$ patients $(n=8)$ with T-ALL achieved a complete remission with or without hematopoietic recovery with another $31 \%$ of patients $(n=4)$ diagnosed with T-LBL achieved a complete remission. ${ }^{15}$ A total of 28 patient had received at least 2 prior induction regimens before receiving nelarabine. Of these 28 patients, $36 \%(n=10)$ experienced a complete, incomplete or a partial remission when treated with nelarabine. The remaining patient population $(n=11)$ had received only 1 prior induction regimen, $55 \%(n=6)$ achieved a complete, incomplete or a partial remission when treated with nelarabine. ${ }^{15}$

A total of 10 patients $(26 \%)$ achieved a complete remission for a median duration of 27 weeks. ${ }^{15}$ A total of $41 \%$ $(n=16)$ achieved either a complete or partial remission to treatment with nelarabine. The median survival for patients with 1 prior induction regimen was 20 weeks (95\% CI 12-219 weeks) with the median survival for 2 prior induction regimens as 20 weeks (95\% CI 10 to 36 weeks). ${ }^{15}$ Based upon the response in this trial the investigators concluded that the 1-year disease-free survival for all patients was $28 \%{ }^{15}$

The National Cancer Institute sponsored a Phase II study at MD Anderson Cancer Center (MDACC) which evaluated nelarabine therapy in patients diagnosed with non-Hodgkin's lymphoma. The study population consisted of 23 patients, 17 males and 6 females, within the age range of 33 to 81 years. ${ }^{19}$ The patient population consisted of 13 patients diagnosed with peripheral T-cell lymphoma and 10 patients diagnosed with low-grade B-cell lymphoma and both groups were considered relapsed and/or refractory secondary to failure of 1 to 3 previous chemotherapy regimens. ${ }^{19}$ Nelarabine $1.5 \mathrm{~g} / \mathrm{m}^{2}$ was administered intravenously on an alternate day regimen on days 1, 3 and 5 and was repeated every 28 days for a total of 6 cycles. ${ }^{19}$ The study regimen was held in the presence of disease progression or toxicity. The average number of cycles received by the study participants was 3.1 cycles. ${ }^{19}$

Eight out of the 13 patients diagnosed with peripheral T-cell lymphoma were evaluable, and 2 patients in this group died (unrelated to treatment with nelarabine) and 2 patients were in the early stages of the nelarabine regimen and 1 patient was removed from the study secondary to adverse events. ${ }^{19}$ Overall, $50 \%$ of the patients diagnosed with peripheral T-cell lymphoma achieved either a complete $(n=2 / 8)$ or a partial $(n=2 / 8)$ response. ${ }^{19}$ Overall, 9 out of the 10 patients with low-grade B-cell lymphoma a total of $44 \%$ of patients $(n=4)$ experienced a partial response. ${ }^{19}$ The remaining patients either had stable disease or disease progression while receiving nelarabine. ${ }^{19}$

A Phase 2 study was sponsored by The Pediatric Oncology Group/Children's Cancer Group (POG/CCG), which evaluated the response to nelarabine in patients with 
refractory T-cell hematologic malignancies or non-Hodgkin's lymphoma. The study population consisted of children $(<21$ years of age) with refractory or recurrent T-cell acute lymphoblastic leukemia or non-Hodgkin's lymphoma. ${ }^{20}$ This study population was stratified into 1 of 4 groups based upon the following classification: 1) patients refractory to initial induction therapy or $\geq 25 \%$ bone marrow blasts in first relapse; 2) patients with $\geq 25 \%$ bone marrow blasts in second or greater relapse; 3) patients with positive cerebrospinal fluid and $\geq 5 \%$ bone marrow blasts; 4 ) patients with extramedullary relapse and $<25 \%$ bone marrow blasts. Nelarabine $1.2 \mathrm{~g} / \mathrm{m}^{2}$ was administered intravenously over 60 minutes for 5 consecutive days every 3 weeks. ${ }^{20}$ This dose was adjusted in Groups 1 and 2 to $650 \mathrm{mg} / \mathrm{m}^{2}$ secondary to neurotoxic adverse events..$^{20}$ The patients in Groups 3 and 4 received nelarabine $400 \mathrm{mg} / \mathrm{m}^{2}$. A total of 153 patients were initially enrolled and 121 were enrolled at the final dose levels and 106 patients were evaluable, with 33 patients in Group 1, 30 patients in group 2, 21 patients in group 3, and 22 patients in group $4 .^{20}$ The responses to nelarabine were as follows: Group 1, 18 patients responded to nelarabine with $48 \%(n=16 / 33)$ achieving a complete response and $6 \%$ $(\mathrm{n}=2)$ experienced a partial response; Group 2 had a total of $23 \%$ of patients $(n=7 / 30)$ achieving a complete response and $3 \%(n=1 / 30)$ had a partial response; Group 3, overall $24 \%(\mathrm{n}=5 / 21)$ experienced a complete response and $10 \%$ $(n=2 / 20)$ had a partial response; Group 4, 14\% $(n=3 / 22)$ achieved a partial response to treatment with nelarabine. ${ }^{20}$

This Phase 2 study clearly demonstrated that nelarabine is a viable treatment option for patients diagnosed with relapsed or refractory T-cell malignancies, with the strongest clinical results found in patients with T-cell leukemia in first relapse. ${ }^{20}$ Overall, of the 106 patients evaluated, 26\% $(\mathrm{n}=28 / 106)$ had a complete response and $8 \%(\mathrm{n}=9 / 106)$ had a partial response..$^{20}$ Of note, a total of 8 out of 22 patients that had a positive lumbar puncture for CSF disease prior to nelarabine administration had negative lumbar puncture for CSF disease only 7 days after the initiation of nelarabine treatment regimen. These responses occurred prior to any intrathecal treatment. ${ }^{20}$

\section{Safety}

Overall tolerability of nelarabine during a phase I trial by Kurtzberg et al was related to dose limiting neurotoxicity that was often reversible upon drug discontinuation or dose reduction. The incidence of neurologic toxicity was correlated to the dose of nelarabine received with more severe neurotoxicity occurring more frequently in patients receiving higher doses of nelarabine. ${ }^{18}$ However the severity of the neurotoxicity experienced cannot be reliably correlated with the dose of nelarabine administered, as adult and pediatric patients receiving modest doses $(10 \mathrm{mg} / \mathrm{kg} /$ day and $20 \mathrm{mg} / \mathrm{kg} /$ day) experienced similar neurotoxicity such as somnolence, malaise and fatigue, as adults and pediatric patients receiving higher doses of nelarabine $(40 \mathrm{mg} / \mathrm{kg} /$ day and $60 \mathrm{mg} / \mathrm{kg} / \mathrm{day}){ }^{18}$

Neurologic adverse events directly attributable to nelarabine occurred in 72\% $(n=67 / 93)$ patients enrolled in this phase I trial. ${ }^{18}$ Approximately half of the children enrolled and $85 \%$ of the adults experienced reversible neurotoxicity secondary to receiving the study drug. ${ }^{18}$ Symptom presentation began with transient somnolence, malaise with overt fatigue predominantly occurring 6 to 8 days after the initiation of the nelarabine regimen, while the onset of neurotoxicity symptoms generally appeared 12 days post-regimen initiation. ${ }^{18}$ Both central and peripheral toxicities were reported. While there were no documented delayed or latent neurotoxicities observed the study was not designed for follow-up in terms of medication-related toxicities. ${ }^{18}$

A total of 11 patients treated with higher doses of nelarabine $\left(1.2 \mathrm{~g} / \mathrm{m}^{2}\right.$ or $40 \mathrm{mg} / \mathrm{kg}$ and $60 \mathrm{mg} / \mathrm{kg}$ in adult and pediatric patients, respectively) experienced cumulative neurotoxicity depicted as hypoesthesias, paresthesias or peripheral neuropathies. ${ }^{18}$ Within this group $3 / 11$ patients experienced a severe grade of neurotoxicity with 4/11 remaining unresolved at the end of the study. One pediatric patient diagnosed with T-cell acute lymphoblastic leukemia in refractory relapse experienced severe and irreversible neurotoxicity after receiving treatment with $75 \mathrm{mg} / \mathrm{kg} /$ day of nelarabine. ${ }^{18}$ Approximately 12 days after initiating the treatment regimen this pediatric patient experienced seizures, myoclonic jerks, ascending paralysis and coma resulting eventually in death. The overall neurotoxicity was extensive, requiring mechanical ventilation and the patient did not regain full consciousness.

Although a significant portion of patients enrolled in the phase I trial experienced dose limiting neurotoxicity, the patients had concomitant factors for the development of neurotoxicity. ${ }^{18}$ Of these 11 patients treated with higher doses of nelarabine, 8 had received prior therapy with vincristine which could have contributed to the development of neurotoxicity. ${ }^{18}$ However, 70 other patients within the study had received prior therapy with vincristine $(>1$ month prior to study enrollment) and did not develop cumulative neurotoxicity. Furthermore, $73 \%$ of patients previously 
treated with high dose cytarabine, $71 \%$ previously treated with vincristine, $60 \%$ having received intrathecal therapy, $53 \%$ with prior radiation therapy and $67 \%$ with presence of leukemic disease in the CSF at the time of treatment all experienced neurotoxicity independent of the dose of nelarabine administered.$^{18}$ In addition, all patients of at least 60 years of age experienced neurotoxicity.

Evaluating the hematologic impact of nelarabine, a total of $77(83 \%)$ patients had documented disease in the bone marrow upon entry into the study and made hematologic toxicities difficult to accurately quantify. ${ }^{18}$ Hematologic toxicities observed in the phase I trial by Kurtzberg et al included Grade 1 and 2 neutropenia and thrombocytopenia in $50 \%$ and $76 \%$ of pediatric patients, respectively and $46 \%$ and $42 \%$ of adult patients, respectively. In patients who achieved a documented complete response or had no documented bone marrow involvement, there was no Grade 3 or 4 hematologic toxicities. ${ }^{18}$ At the conclusion of the study, hematologic toxicities were determined not to be cumulative or dose limiting.

The most common nonneurologic and nonhematologic toxicities encountered affected $70 \%$ of the study population and included nausea, vomiting, diarrhea, fever, and anorexia. ${ }^{18}$ The occurrences of these toxicities were observed more frequently in adults compared to children. Complications secondary to these toxicities were not noted in any adult or pediatric patient. ${ }^{18}$

In the CALGB phase II trial safety and toxicity information was available for 38 of the 39 patients enrolled in the study. ${ }^{15} \mathrm{~A}$ total of 14 patients $(37 \%)$ experienced grade 1 or 2 peripheral sensory neuropathy and 8 patients (21\%) experienced peripheral motor neuropathy, only 1 case being identified as grade $3 .^{15}$ Two patients withdrew from the study after experiencing grade 3 and 4 peripheral sensory neuropathy. ${ }^{15}$ During the study 1 patient who had been treated with a cumulative dose of $4.5 \mathrm{~g} / \mathrm{m}^{2}$ of nelarabine experienced a grade 3 seizure; however this event was thought to be secondary to imipenem and renal dysfunction. ${ }^{15}$ There was only 1 case of a grade 4 neurologic toxicity which was noted as a reversible depressed level of consciousness. No patient deaths were linked to the protocol or treatment with nelarabine. ${ }^{15}$

Nonhematologic events encountered during the CALGB trial were classified as a grade 3 or higher toxicity and included fatigue $(n=7)$ and muscle weakness $(n=4) .{ }^{15}$ Gastrointestinal complaints were noted in $51 \%$ of the patients and included nausea, vomiting, diarrhea, and stomatitis, however only one of the events was classified as severe (grade 3 diarrhea). ${ }^{15}$ Hematologic toxicities encountered were grade 3 and 4 and were predominantly neutropenia and thrombocytopenia. Grade 3 neutropenia was experienced by a total of 5 patients (13\%) with 11 patients classified with grade 4 neutropenia. ${ }^{15}$ Thrombocytopenia of grade 3 and 4 was experienced by 7 patients $(18 \%)$ and 6 patients $(16 \%)$, respectively. ${ }^{15}$

Adverse events encountered in the MDACC study also included neurotoxicities secondary to nelarabine administration. Out of 23 patients enrolled, there were 2 grade 3/4 neurotoxic adverse events. One patient experienced sensory neuropathy following the sixth cycle of nelarabine, and the second patient developed ascending sensory and motor polyneuropathy during the initial cycle of therapy. ${ }^{19}$ Additionally, one other patient experienced central nervous system toxicity, described as acute onset of confusion which resolved within 48 hours. Throughout the remainder of the study there were no other high grade or intolerable adverse events noted. The only additional adverse events encountered included a grade 3 myalgia $(n=1)$, grade $3 / 4$ infection $(n=3)$ and grade 3 diarrhea $(\mathrm{n}=1)$. For hematologic toxicities, 1 patient had neutropenia and 1 patient had grade 3 thrombocytopenia. ${ }^{19}$

The POG/CCG trial also encountered neurotoxic adverse effects secondary to treatment with nelarabine. All children who received at least 1 dose of nelarabine were evaluated for potential neurotoxic adverse effects $(n=151) .{ }^{20} \mathrm{~A}$ total of $18 \%(n=27)$ of enrolled patients experienced either a grade 3 or 4 neurotoxicity, although not all events were entirely attributable to the administration of nelarabine. ${ }^{20}$ Patients that received nelarabine $400 \mathrm{mg} / \mathrm{m}^{2} /$ day for 5 consecutive days $(n=50)$ resulted in a total of 17 grade $3 / 4$ neurologic toxicities, 6 being peripheral in origin. ${ }^{20}$ Patients that received nelarabine $650 \mathrm{mg} / \mathrm{m}^{2} /$ day for 5 consecutive days (84) resulted in 10 patients experiencing a grade $3 / 4$ neurologic toxicity. ${ }^{20}$ Finally, 4 patients receiving nelarabine $900 \mathrm{mg} / \mathrm{m}^{2} /$ day for 5 consecutive days experienced a grade $3 / 4$ neurologic toxicity and 2 grade $3 / 4$ peripheral nervous system toxicity. Tables 2 and 3 contain cumulative data presented by the US FDA Oncology Drugs Advisory Committee (ODAC) regarding the prevalence of neurotoxicity in patients treated with nelarabine.

The potential neurotoxicity induced by nelarabine appears to have both modest and severe symptom presentation and has been reported as reversible in several cases. ${ }^{15,17,18}$ Patients who experienced the neurotoxic effect often had received prior therapy with a different chemotherapeutic agent that also has the potential to induce neurotoxicity, and the status 
Table 2 Neurologic adverse events in pediatric patients treated with nelarabine $650 \mathrm{mg} / \mathrm{m}^{2}$ intravenously for 5 consecutive days, repeated every 21 days ${ }^{21}$

\begin{tabular}{lll}
\hline Nervous system disorders & \multicolumn{2}{l}{ Patient population $(\mathbf{n}=\mathbf{8 4})$} \\
\cline { 2 - 3 } & Grade 3/4 (\%) & All Grades (\%) \\
\hline Headache & 6 & 17 \\
Peripheral neurologic disorders & 7 & 12 \\
Lowered consciousness & 2 & 7 \\
Peripheral neurologic disorders & 7 & 12 \\
Hypoesthesia & 4 & 6 \\
Seizures & 6 & 6 \\
Motor dysfunction & 1 & 4 \\
Nervous system disorder & 0 & 4 \\
Paresthesia & I & 4 \\
Tremor & 0 & 4 \\
Ataxia & $\mathrm{I}$ & 2 \\
\hline
\end{tabular}

of the patients hematologic malignancy was either relapsed or refractory prior to treatment with nelarabine. ${ }^{15,18}$ For all therapeutic modalities the risk versus the benefit of every option should be considered prior to therapeutic initiation.

\section{Future research}

Nelarabine has been primarily evaluated in adult and pediatric patients diagnosed with T-cell lymphoblastic lymphoma or leukemia whose disease is refractory or has relapsed while receiving treatment with other chemotherapeutic regimens.

Table 3 Neurologic adverse events in adult patients treated with nelarabine $1500 \mathrm{mg} / \mathrm{m}^{2}$ intravenously over 2 hours on days I, 3, and 5 repeated every 21 days $^{21}$

\begin{tabular}{lll}
\hline Nervous system disorders & \multicolumn{2}{l}{ Patient population $(\mathbf{n}=103)$} \\
\cline { 2 - 3 } & Grade 3/4 (\%) & All Grades $(\%)$ \\
\hline Lowered consciousness & 14 & 63 \\
Dizziness & 0 & 21 \\
Peripheral neurologic disorders & 2 & 18 \\
Hypoesthesia & 2 & 17 \\
Headache & 1 & 15 \\
Paresthesia & 0 & 15 \\
Ataxia & 2 & 9 \\
Tremor & 0 & 5 \\
Neuropathy & 0 & 4 \\
Amnesia & 0 & 3 \\
Dysguesia & 0 & 3 \\
Balance disorder & 0 & 2 \\
Sensory loss & 0 & 2 \\
Seizures & 1 & 1 \\
\hline
\end{tabular}

The potential role of nelarabine as a first-line option in patients diagnosed with T-cell malignancies has not been extensively evaluated and presents an opportunity for future research and development.

Current clinical trials have evaluated the safety and efficacy of nelarabine as a single agent administered as monotherapy. Although nelarabine was studied in combination with fludarabine as a potential option to increase intracellular concentrations of the clinically active moiety, ara-GTP, combination with other chemotherapeutic agents has not been extensively evaluted. ${ }^{2}$ Combination or substitution of nelarabine in chemotherapy regimens that contain nucleoside analogs are potential opportunities of evaluation. ${ }^{15,16}$

\section{Conclusion}

Nelarabine, a nucleoside analog, has been approved for the treatment of adult or pediatric T-cell lymphoblastic leukemia or lymphoma that is relapsed or refractory to two or more chemotherapeutic regimens. As demonstrated in clinical trials, nelarabine has proven to be a viable option for patients who have failed other chemotherapeutic regimens. Nelarabine has a distinct role and place in current therapy, and with further research and investigation may also have a role in combination with other chemotherapy.

\section{Disclosures}

The authors declare no conflicts of interest.

\section{References}

1. Pui CH, Robison LL, Look AT. Acute lymphoblastic leukemia. Lancet. 2008;371:1030-1043.

2. Esparza S. Topics in pediatric medicine-acute lymphoblastic leukemia Med Gen Med [PMC1681386]. 2005 Feb [cited 2009 March 10]; 7(1):[about 1p.]. http://www.pubmedcentral.nih.gov/articlerender. fcgi?artid $=1681386$

3. Song KW, Barnett MJ, Gascoyne M, et al. Primary therapy for adults with T-cell lymphoblastic lymphoma with hematopoietic stem-cell transplantation results in favorable outcomes. Ann Oncol. 2007;18:535-540.

4. Getting the Facts: T-cell Lymphomas. Lymphoma Research Foundation; 2008 March:1-4.

5. Gandhi V, Plunkett W, Weller S, et al. Evaluation of the combination of nelarabine and fludarabine in leukemias: clinical response, pharmacokinetics, and pharmacodynamics in leukemia Cells. J Clin Oncol. 2001;19:2124-2152

6. Kurtzberg J. Guanine arabinoside as a bone marrow purging agent. Ann NY Acad Sci. 1993;658:225-236.

7. Gravatt LC, Chaffee S, Hebert ME, et al. Efficacy and toxicity of 9-beta-D-arabinofuranosylguanine ( $\mathrm{araG}$ ) as an agent to purge malignant T-cells from murine bone marrow: Application to an in vivo T-leukemia model. Leukemia. 1993;7:1261-1267.

8. Kisor D, Plunkett W, Kurtzberg J, et al. Pharmacokinetics of nelarabine and 9-beta-D-arabinofuranosyl guanine in pediatric and adult patients during a phase I study of nelarabine for the treatment of refractory hematologic malignancies. J Clin Oncol. 2000;18:995-1003. 
9. www.pbm.va.gov [homepage on the Internet]. National PBM Monograph: Nelarabine (Arranon $^{\circledR}$ ). [updated 2007 Jan; cited 2009 March 10]. http://www.pbm.va.gov/Clinical\%20Guidance/Drug\%20Monographs/ Nelarabine.pdf

10. DeAngelo D. The treatment of adolescents and young adults with acute lymphoblastic leukemia. Hematology Am Soc Hematol Educ Program. 2005:123-130.

11. Rodriquez CO, Mitchell BS, Ayres M, Eriksson S, Ghandi V. Arabinosyl-guanine is phosphorylated by cytoplasmic deoxycytidine kinase and mitochondrial deoxyguanosine kinase. Cancer Res. 2002;62:3000-3005.

12. Rodriguez CO, Ghandi V. Arabinosylguanine-induced apoptosis of T-lymphoblastic cells: incorporation into DNA is a necessary step. Cancer Res. 1999;59:4937-4943.

13. Rodriguez CO, Stellrecht CM, Gandhi V. Mechanism for T-cell selective cytotoxicity of arabinosylguanine. Blood. 2003;102:1842-1848.

14. Ghandi V, Plunkett W, Rodriguez CO, et al. Compound GW506U78 in refractory hematologic malignancies: Relationship between cellular pharmacokinetics and clinical response. J Clin Oncol. 1998;16:3607-3615.

15. DeAngelo DJ, Yu D, Johnson JL, et al. Nelarabine induces complete remission in adults with relapsed or refractory $\mathrm{T}$-lineage acute lymphoblastic leukemia or lymphoblastic lymphoma: Cancer and Leukemia Group B study 19801. Blood. 2007;109:5136-5142.
16. Rodriguez CO, Legha JK, Estey E, et al. Pharmacological and biochemical strategies to increase the accumulation of arabinofuranosylguanine triphosphate in primary human leukemia cells. Clin Cancer Res. 1997; 3:2107-2113.

17. Gandhi V, Tam C, O'Brien, et al. Phase I trial of nelarabine in indolent leukemias. J Clin Oncol. 2008;26:1098-1105.

18. Kurtzberg J, Ernst TJ, Keating MJ, et al. Phase I study of 506U78 administered on a consecutive 5-day schedule in children and adults with refractory hematologic malignancies. J Clin Oncol. 2005; 23:3396-3403.

19. Goy A, Bleyer A, Hagemeister F, et al. Phase II study of compound GW506U78 (araG) for patients with indolent B-cell or peripheral T-cell lymphoma previously treated with chemotherapy. Proceedings of the 45th Annual Meeting American Society of Hematology; 2003; San Diego, U S A. Abstract 2359.

20. Berg SL, Blaney SM, Devidas M, et al. Phase II study of nelarabine (compound 506U78) in children and young adults with refractory T-cell malignancies: A report from the Children's Oncology Group. J Clin Oncol. 2005;23:3376-3382.

21. www.fda.gov [homepage on the Internet]. Questions for the Oncology Drug Advisory Committee; [2005 Sept 14; cited 2009 March 10]. http://www.fda.gov/ohrms/dockets/ac/05/questions/2005-4174Q2_02_ Arranon.pdf
OncoTargets and Therapy

\section{Publish your work in this journal}

OncoTargets and Therapy is an international, peer-reviewed, open access journal focusing on the pathological basis of all cancers, potential targets for therapy and treatment protocols employed to improve the management of cancer patients. The journal also focuses on the impact of management programs and new therapeutic agents and protocols on

\section{Dovepress}

patient perspectives such as quality of life, adherence and satisfaction The manuscript management system is completely online and includes a very quick and fair peer-review system, which is all easy to use. Visit http://www.dovepress.com/testimonials.php to read real quotes from published authors. 